\title{
David McKee, Non ora, Bernardo!, Milano, Mondadori, 2019.
}

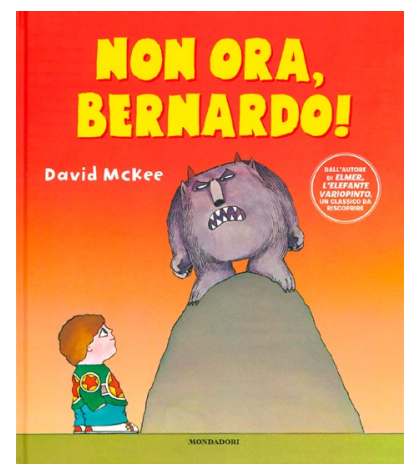

Era il 1980 quando venne pubblicato in traduzione italiana l'albo illustrato Not now, Bernard dell'autore inglese David McKee, la cui edizione originale era uscita nello stesso anno per i tipi della Andersen Press. Il titolo scelto da Rosellina Archinto, fondatrice della Emme Edizioni di Milano (una casa editrice che, per le coraggiose scelte di rottura con la tradizione dei libri per l'infanzia in lingua italiana, rivoluzionò il mercato editoriale del settore), non ne rappresentava certo una versione letterale, ma ne esaltava il succo senza giri di parole: Non rompere, Giovanni. La natura "divergente” della scelta apparirà chiara ripercorrendo la storia, che è molto semplice: un bambino, Bernard, cerca di attirare l'attenzione dei suoi genitori, ma inutilmente, perché loro, presi in occupazioni domestiche banali, ogni volta gli rispondono con il "ritornello" del titolo originale («Not now, Bernard»), che nella versione italiana risultava dunque ancora più forte (appunto «Non rompere, Giovanni»). Il bambino allora si reca in giardino, dove c'è un minaccioso mostro che, senza indugi, se lo mangia, restando poi soddisfatto a contemplare una sua scarpina. Il mostro, poi, entra in casa e minaccia madre e padre, con il medesimo risultato che aveva ottenuto, con loro, il bambino: «Not now, Bernard». I genitori neppure si accorgono che il loro piccolo Bernard è stato sostituito da un mostro; addirittura, la mamma giunge 
al punto di mettere a letto il mostro, dandogli la buonanotte con il consueto "ritornello". E così finisce la storia. Niente lieto fine, niente riconciliazione, ma uno strappo netto, definitivo, irrimediabile.

Si capisce dunque perché il libro ebbe un impatto forte sul mercato e, più ancora, sulla sensibilità comune, ancora calibrata sui caratteri che la letteratura per l'infanzia in lingua italiana aveva ereditato dalla produzione classica di epoca ottocentesca, cioè l'intento pedagogico quasi sempre esplicitamente edificante. E forse si possono intuire anche i motivi, prettamente editoriali, nascosti dietro la successiva "sparizione" dell'albo dai cataloghi di vendita, dopo l'esaurimento della prima edizione.

Oggi, però, l'albo illustrato di David McKee è di nuovo disponibile anche per i lettori italiani, grazie alla nuova edizione della Mondadori, che si mostra maggiormente fedele all'originale sin dal titolo, che ne è una traduzione letterale: Non ora, Bernardo! (con la sola aggiunta del punto esclamativo). Ma perché bisogna essere grati alla Mondadori per questa iniziativa? Prima di tutto perché anche i bambini hanno i loro problemi, e non sono solo questioni di giocattoli e di cartoni animati: molti di loro sono turbati da pensieri che li inquietano, che minano la loro naturale spensieratezza. E a volte, per farli riflettere, per far sì che questi problemi possano essere meglio definiti, e magari risolti, può essere opportuno che i bambini si trovino confrontati con essi, in modo "provocatorio", cioè per "tirare fuori la loro voce”, e non sempre e solo attraverso storie edificanti e a lieto fine, anche perché nella vita non sempre tutto va a finire per forza bene. A volte, infatti, i problemi sembrano avere il sopravvento. Ma un primo passo per iniziare a superarli è prendere coscienza che essi ci sono, e che vanno affrontati, ad esempio parlandone. Ed è proprio qui che un libro come quello di David McKee può entrare in gioco: perché offre l'occasione per dare voce ai problemi proprio ai bambini che li hanno, e che magari non hanno il coraggio di condividerli.

Il libro, infatti, parla di uno dei nodi cruciali nella vita di ogni bambino, cioè il rapporto con i genitori: sereno, a volte, ma molto spesso problematico, fonte di conflitti e di turbamenti, quando non di dolore e terrore. Ecco che l'albo diventa un modo per parlare tutti insieme di un problema che più o meno tutti i bambini si trovano ogni tanto ad affrontare: quello dei genitori che "non hanno 
tempo", presi nelle loro occupazioni e persi nei loro pensieri. La discussione diventa dunque un modo per capire meglio il punto di vista altrui (dei genitori e del bambino), al fine di esorcizzare una delle più grandi paure: quella di non essere ascoltati, fino a non esistere. Per arrivare, magari e solo in un secondo tempo, a una riscrittura o a una rivisitazione della storia di partenza, a partire da stimoli come "Ma siamo proprio sicuri che il mostro abbia mangiato Bernardo? Non è che per caso lui è fuggito, e ha perso una scarpina?"; oppure "Proviamo a inventare un nuovo finale a questa storia, un finale in cui ci sia ancora Bernardo"; oppure ancora "Immaginiamo che cosa sarebbe potuto succedere se il papà o la mamma di Bernardo lo avessero ascoltato, invece di dirgli non ora, Bernardo”. Possiamo arrivare anche alla rappresentazione teatrale, basata su un copione ispirato alla storia, in cui i bambini impersonano bambini e genitori che non li ascoltano. Insomma, l'importante è non lasciare il bambino da solo con questa lettura, altrimenti rischieremmo di comportarci più o meno come i genitori della storia: il libro deve essere uno spunto per riflettere, per approfondire, per poi andare oltre.

Simone Fornara 